\title{
Noise-induced Brownian motion of spiral waves
}

\author{
S. Alonso ${ }^{1,2, *}$ and F. Sagués ${ }^{1}$ \\ ${ }^{1}$ Departament de Química Física, Universitat de Barcelona, Avenida Diagonal 647, 08028 Barcelona, Spain \\ ${ }^{2}$ Departament d'Estructura i Constituents de la Matèria, Universitat de Barcelona, Avenida Diagonal 647, 08028 Barcelona, Spain
}

(Received 17 November 2000; published 22 March 2001)

\begin{abstract}
We study the erratic displacement of spiral waves forced to move in a medium with random spatiotemporal excitability. Analytical work and numerical simulations are performed in relation to a kinematic scheme, assumed to describe the autowave dynamics for weakly excitable systems. Under such an approach, the Brownian character of this motion is proved and the corresponding dispersion coefficient is evaluated. This quantity shows a nontrivial dependence on the temporal and spatial correlation parameters of the external fluctuations. In particular, a resonantlike behavior is neatly evidenced in terms of the noise correlation time for the particular situation of spatially uniform fluctuations. Actually, this case turns out to be, to a large extent, exactly solvable, whereas a pair of dispersion mechanisms are discussed qualitatively and quantitatively to explain the results for the more general scenario of spatiotemporal disorder.
\end{abstract}

DOI: 10.1103/PhysRevE.63.046205

PACS number(s): 82.40.Bj, 05.40.-a

\section{INTRODUCTION}

Spiral excitation waves are among the most paradigmatic examples of spatiotemporal self-organizing structures in nonequilibrium extended systems [1]. They have been observed in very different systems, ranging from the prototypical Belousov-Zhabotinsky (BZ) reaction [2] or catalytic surface processes [3], to aggregating colonies of social amoebae and other microorganisms [4] or propagating patterns of heart tissue excitations [5]. Given the wide variety of scenarios just mentioned, one could easily admit that spiral formation and propagation could likely take place in many instances under different forcing conditions or spatiotemporal heterogeneities.

Actually, the response of spiral waves to spatial and/or temporal forcing of excitable systems has been widely analyzed in the literature. Temporal forcing [6,7], drift of vortices due to parameter gradients [8] or external fields [9] are among the most studied effects. More recently, feedbackbased forcing schemes have been also considered $[10,11]$. In addition, either propagation modes or interaction conditions for spirals in heterogeneous media have been also examined from different perspectives [12-15] [see also Ref. [16] by Hendrey et al., although referring this time to complex Ginzburg-Landau (CGL) spirals for oscillatory media].

On the other hand, the role of random heterogeneities on extended excitable systems has recently attracted much attention. Noise as an initiator of new spatial structures [1719], or sustaining wave propagation in subexcitable media [20-22], is a subject of much theoretical and experimental interest. Complementarily, the role of superimposed disorder on preexisting spatiotemporal patterns has been examined in relation to propagating pulses [23], CGL spirals [24], and three-dimensional structures [25].

As a direct antecedent of what is going to be presented here, we recently reported [26] on experimental, numerical, and analytical work conducted to investigate the response of

\footnotetext{
*Corresponding author. Email address: s.alonso@qf.ub.es
}

spiral waves to structured noise. The particular system chosen corresponds to the photosensitive BZ reaction under spatiotemporal fluctuations in the illumination. In the absence of randomness, the initial configuration was that of a steadily rotating spiral with no net translational motion. When the noise was switched on, Brownian wandering of the spiral tip was evidenced and characterized in terms of a diffusion coefficient, showing a nonmonotonic dependence on the parameters of the noise [see Fig. 1(a)]. In particular, perturbing the spiral with fixed noise dispersion and varying its time correlation, a resonantlike effect was observed for pure spatially uniform fluctuations, leading to a neat enhancement of the diffusion coefficient when the correlation time matched the intrinsic rotation frequency of the spiral. In the more general case corresponding to a forcing pattern of spatiotemporal random illumination, absolute values of the dispersion coefficient were even larger, featuring again maxima which appeared this time shifted to larger correlation times. These observations were confirmed numerically using a twovariable Oregonator model [see Fig. 1(b)] [26]. In addition, a theoretical formulation, based on a kinematic approach to the spiral dynamics, was proposed that reproduced the basic features observed in the experiments and numeric simulations.

Here, continuing that research, we present a more exhaustive analytical and numerical study of that phenomenon.
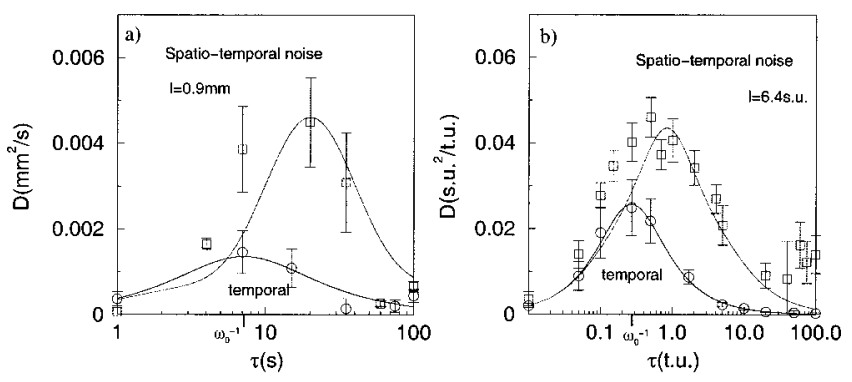

FIG. 1. Experimental and numeric results for the effective diffusion coefficient $D$ vs $\tau$. (a) Experimental results for the BelousovZhabotinsky photosensitive reaction. (b) Numeric results for the two-variable Oregonator model. See Ref. [26] for details. 
Again the theoretical framework we will adopt is that of a kinematic approach valid for weakly excitable systems (sparse spirals) [27]. This approach essentially aims at simplifying the whole description of the extended spiral structure to the dynamics of a single smooth oriented curve whose motion is described by appropriate equations written for its normal and tangential propagation velocities. Later on, such a phenomenological approach is further reduced to the description of a pointlike object representing the tip of the spiral wave. Proceeding in this way, the whole dynamics is parametrized in terms of a set of kinematic quantities, which incorporate the excitability dependent propagation conditions of the medium where spiral propagation takes place. External spatiotemporal fluctuations with well-defined statistics and correlations are then introduced through such a set of basic kinematic parameters. To be more specific, we consider Gaussian, zero mean Ornstein-Uhlenbeck cell distributed fluctuations, changing continuously with time and independently from cell to cell. In what follows we report on a series of results organized in such a way that both the noise dispersion and the correlation length are essentially fixed, while varying the correlation time. In particular, the cell dimension, essentially a measure of the spatial correlation of the fluctuations, is assumed to be always comparable to or larger than the size of the spiral core. For the sake of comparison, we also include results with pure temporal random forcing (spatially uniform fluctuations), corresponding to the limit of infinite correlation length.

Motivated indeed by the experimental observations on the BZ medium we recalled above, but recognizing at the same time the difficulty in running such experiments under the somewhat ideal conditions appropriate to the kinematic approach as formulated in Ref. [27], we do not aim this time at either a direct comparison or a fitting of our results with those of the experiments. Rather, we prefer to consider the kinematic equations, appropriate to the spiral motion for weakly excitable systems, as objects of analytic and numeric research by themselves, and undertake a study of their response to spatiotemporal external noise. This notwithstanding, and as could be expected from what was reported in Ref. [26], the most distinctive features of randomly forced spirals, i.e., the resonantlike behavior for pure temporal noise and the enhancement of spiral Brownian motion under spatiotemporal disorder, are consistently reproduced by the stochastic version of the kinematic model, and both effects are indeed largely amenable to a theoretical description as explained here.

The organization of the paper is as follows. In Sec. II we reproduce, for the sake of self-completeness of the paper, the basic set of kinematic equations as they are formulated in Ref. [27]. Sections III and IV are devoted to specifying the numerical procedures and noise prescriptions used throughout the paper. In particular, this is necessary to adapt the spatially discretized noise parametrization used in the numerical work to the continuous one appropriate to the analytical description. Section V is fully dedicated to the situation of spatially uniform fluctuations (pure temporal noise). The analytical treatment to calculate a diffusion coefficient is sketched there, together with the results of some numerical simulations of the stochastic kinematic equations. For the sake of clarity, the material is divided into different sections organized with increasing analytical complexity depending on the assumed level of random parametric forcing. Section VI contains analogous results for spatiotemporal fluctuations. There we first present separately, in two sections, the cases of random effects, introduced through the normal and tangential velocities of the spiral wave at its tip, reserving the last section for the treatment of the fully stochastic equations. Finally, Sec. VII is devoted to summarizing our results and drawing some conclusions.

\section{KINEMATIC MODEL FOR TWO-DIMENSIONAL SPIRAL WAVES}

Within this approach, we start by invoking the so-called quasistatic approximation, which allows us to reduce further the dynamics of a spiral wave to the motion of its tip [27]. In a Cartesian coordinate frame such equations are written as

$$
\begin{gathered}
\frac{d X}{d t}=-V \sin \alpha-G \cos \alpha, \\
\frac{d Y}{d t}=V \cos \alpha-G \sin \alpha,
\end{gathered}
$$

where $X$ and $Y$ are the tip coordinates, $\alpha$ its angular variable, and $V$ and $G$ denote, respectively, the normal and tangential (respective to the spiral curve) tip velocities. These two quantities depend on curvature effects, parametrized by the instantaneous spiral curvature at the tip denoted $k$, and are, respectively, expressed as

$$
\begin{gathered}
V=V_{0}-D k, \\
G=\gamma\left(k_{c}-k\right),
\end{gathered}
$$

in terms of the set of basic kinematic parameters $V_{0}, k_{c}, D$, and $\gamma$. Under the limit of weak excitability, implicitly assumed on all of what follows, the curvature correction to the normal velocity will be assumed negligible $\left(D k \ll V_{0}\right)$, so that $V$ reduces to $V_{0}$, the excitability dependent parameter denoting the intrinsic propagation velocity of uncurved waves.

The particular solution corresponding to a rigid rotating spiral can be simply recovered by taking $G=0$, i.e., $k_{c}=k$, and putting $\alpha=\omega_{0} t$, in terms of the pair of related and intrinsic kinematic parameters $k_{c}$, the critical curvature, and $\omega_{0}$, the natural frequency of the rotating spiral [27],

$$
\omega_{0}=\zeta\left(D V_{0}\right)^{1 / 2} k_{c}^{3 / 2}
$$

with $\zeta$ being a numeric constant: $\zeta=0.685$.

In general, to obtain solutions for quasirigid rotating spirals, we need to supplement Eqs. (1)-(2) with appropriate equations for $\alpha$ and $k$, both being assumed to be timedependent quantities. These equations read

$$
\frac{d k}{d t}=-\frac{1}{\tau_{g}}\left(k-k_{c}\right),
$$




$$
\frac{d \alpha}{d t}=\omega+G k
$$

Apart from the characteristic rotation period, Eq. (6) introduces through $\tau_{g}$ a second time scale into the problem, corresponding to the relaxation of the instantaneous tip curvature to its critical value. The parameter $\tau_{g}$ will play an important role in what follows and can be expressed in terms of $\omega_{0}$ as

$$
\tau_{g}=\frac{D}{\gamma \omega_{0}}
$$

In the context of the phenomenological kinematic theory, $D$ and $\gamma$ are not restricted by any inner condition, so that they are normally assumed to be of the same order [27]. This means that the two time scales appearing so far in the theory are comparable. Finally $\omega$ can be formally expressed in terms of $k$ by generalizing Eq. (5) above to

$$
\omega=\zeta\left(D V_{0}\right)^{1 / 2} k^{3 / 2} \text {. }
$$

The set of kinematic equations (1)-(9) is now complete and ready to be formally examined when the intrinsic kinematic parameters are subjected to spatiotemporal fluctuations. In principle, and lacking a more fundamental understanding of the way such parameters really depend on the excitability of the system, one could assume that any of them, i.e., $V_{0}, k_{c}$, and the pair of curvature related coefficients $D$ and $\gamma$, could be sensitive to noise effects. However, the system of kinematic equations is so complicated that simplifications are necessary to render our analysis clear enough to detect neat and systematic effects. In this sense and based on our simulation results, we tend to think that $V_{0}$ and $k_{c}$ are by far the most natural candidates to incorporate parametric noise. In addition, it will be clear in what follows that the role of structured noise superimposed on these two parameters are notoriously distinct and we are confident that they capture the most representative effects of external fluctuations interacting with the spiral wave dynamics. So we generally consider situations with

$$
\begin{gathered}
V_{0}=\bar{V}_{0}+\xi_{V_{0}}, \\
k_{c}=\bar{k}_{c}+\xi_{k_{c}},
\end{gathered}
$$

where $\xi_{V_{0}}$ and $\xi_{k_{c}}$ denote zero-mean, Gaussian, spatiotemporal noises whose statistics are further described in the following sections.

\section{NUMERICAL PROCEDURES}

The numerical simulations have been run with an explicit Euler method for the integration of the equations for the tip coordinates. The time integration step has been chosen as $\Delta t=1$ t.u., whereas the period of the deterministic spiral was, for most of the cases, $T_{0}=290$ t.u. (temporal and spatial units depend on the excitable system where the theory is applied). The total time of simulation has always been sev- eral orders of magnitude larger than the rotation period, typically at least $500 T_{0}$.

Spatiotemporal noise is distributed in square cells or pixels of linear dimension $l$ in a completely uncorrelated way and satisfying individually the Ornstein-Uhlenbeck statistics [28]

$$
\begin{aligned}
\left\langle\xi_{P, i j}(t) \xi_{Q, k l}\left(t^{\prime}\right)\right\rangle & =\sigma_{P Q}^{2} e^{-\left|t-t^{\prime}\right| / \tau} \delta_{i k} \delta_{j l}, \\
\{P, Q\} & =\left\{V_{0}, k_{c}\right\} .
\end{aligned}
$$

In what follows we report on numerical experiments on spiral wave dispersion organized in series corresponding to fixed values of $l$. This quantity, i.e., the noise correlation length, was prescribed always larger than the spiral core $(l$ $>2 R_{0} ; l=100 \mathrm{~s} . \mathrm{u}$. or $200 \mathrm{~s}$.u. for a typical value of $R_{0}$ $=46$ s.u.). In each series we varied the correlation time $\tau$, keeping the noise dispersion $\sigma_{P Q}^{2}$ constant. For the sake of comparison, numerical simulations were also performed with pure temporal (spatially uniform) noise corresponding to the limit of infinite $l$.

The total size of the grid has been chosen one order of magnitude bigger than the spiral core: from 1000 s.u. to 6000 s.u., depending on the dispersion of the spiral. Typically, the number of realizations needed to evaluate the diffusion coefficient range from 300 for uniform fluctuations to 200 for the spatiotemporal noise.

\section{NOISE SPECIFICATIONS}

In the preceding section we have already described numerically the way random fluctuations are introduced into the kinematic scheme. However some further specifications are necessary.

To put our analysis here in the perspective of the experimental work on the BZ illuminated medium, and before starting our simulations with spiral dispersion, we determined numerically the dependence of the two basic kinematic parameters $V_{0}$ and $k_{c}$ on the illumination parameter. The summarized results of such numerical work, based on the two-variable version of the Oregonator model adapted to a light-sensitive BZ medium, convinced us that fluctuations in $V_{0}$ and $k_{c}$ are correlated, the simplest choice being to assume them to be linearly correlated and of similar relative magnitudes [see Fig. 2].

Using the notation introduced previously in Eq. (12), this means that the three corresponding noise dispersions, denoted $\sigma_{V_{0}, V_{0}}^{2}, \sigma_{V_{0}, k_{c}}^{2}$, and $\sigma_{k_{c}, k_{c}}^{2}$ will actually be assumed to be proportional. To simplify the notation we hereafter denote $\sigma_{V_{0}, V_{0}}^{2}$ simply by $\sigma_{V_{0}}^{2}$ and correspondingly $\sigma_{V_{0}, k_{c}}^{2} \equiv \nu \sigma_{V_{0}}^{2}$ and $\sigma_{k_{c}, k_{c}}^{2} \equiv \nu^{2} \sigma_{V_{0}}^{2}$, in terms of an ad hoc proportionality constant denoted $\nu$, to be fixed later on.

The second specification refers to the analytical results that are going to be presented in the next sections. As will become clear in what follows, most of these results do admit elegant formal generalizations beyond the OrnsteinUhlenbeck-like temporal correlation prescribed in all our numerical work according to Eq. (12). This means that analyti- 

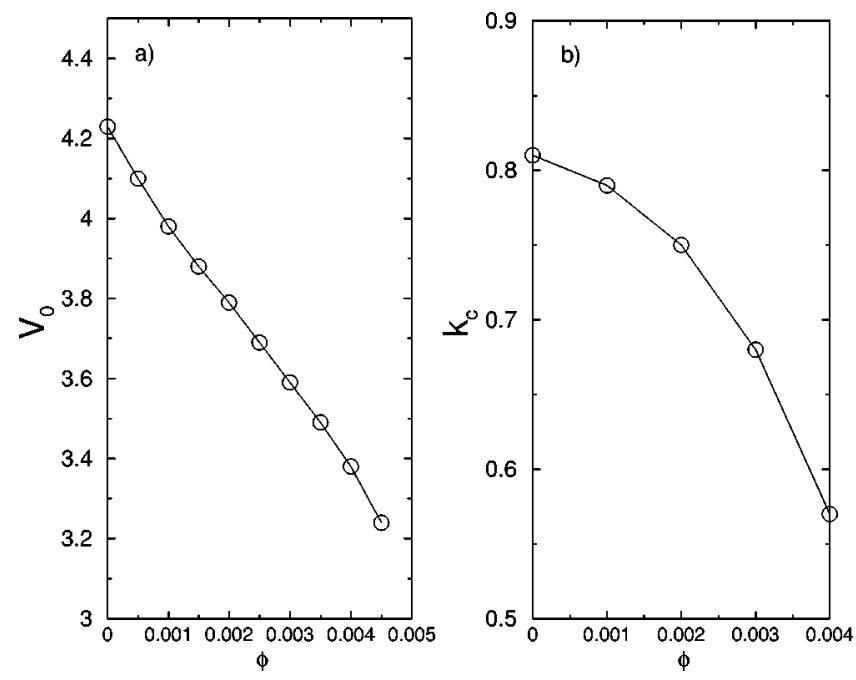

FIG. 2. Results for kinematic parameters $V_{0}$ and $k_{c}$ obtained from numerical simulations of the two-variable reaction-diffusion photosensitive Oregonator model [26] with numerical values of $f=1.4, q=0.002, \epsilon=0.01, D_{u}=1$, and $D_{v}=0.6$ (a) Linear behavior is observed for the normal velocity $V_{0}$ of the spiral versus the illumination parameter $\phi$. (b) Critical curvature $k_{c}$ of the spiral waves versus illumination parameter $\phi$, where the critical curvature has been calculated with the kinematic expression $k_{c}$ $=\left(V_{0} / \zeta^{2} D R_{0}^{2}\right)^{1 / 3}[27]$.

cal results are going to be obtained as much as possible for stationary noises, with generic time correlation denoted as $\left\langle\xi(t) \xi\left(t^{\prime}\right)\right\rangle \propto C_{\xi}\left(\left|t-t^{\prime}\right|\right)$ (spatial dependence is not kept in this expression to simplify notation and because it is not going to be explicitly necessary in what follows). Obviously, when we compare analytical expressions with numerical results, we will adapt our formal scheme to the OrnsteinUhlenbeck prescription by replacing $C_{\xi}\left(\left|t-t^{\prime}\right|\right)$ by $\sigma^{2} e^{-\left|t-t^{\prime}\right| / \tau}$.

The final necessary specification refers to the way the discrete spatial nature of the noise in the numerical simulations Eq. (12) is translated into our space-continuous phenomenological equations. Actually, we had several options that should be conveniently discussed. In any case, the necessary condition to be fulfilled is the statistical independence of the temporal and spatial correlation components

$$
\left\langle\xi(\mathbf{r}, t) \xi\left(\mathbf{r}^{\prime}, t^{\prime}\right)\right\rangle=C_{\xi}\left(\left|t-t^{\prime}\right|\right) C\left(\mathbf{r}-\mathbf{r}^{\prime}\right) .
$$

The obvious choice is to prescribe a triangularlike form for the spatial part, i.e.,

$$
C\left(\mathbf{r}-\mathbf{r}^{\prime}\right)=\left(1-\frac{\left|x-x^{\prime}\right|}{l}\right)\left(1-\frac{\left|y-y^{\prime}\right|}{l}\right)
$$

This correlation function is anisotropic and the calculations with it become very complicated. Other simpler choices that we expect to give qualitatively similar but simpler results arise from the following argument. Since diffusion is a process that is related to long times and large distances, the behavior of the system should be independent of the local topology of the system while keeping the characteristic cor- relation length. So we can enforce the isotropic character of the correlation function with different forms like the one adopted here,

$$
C\left(\left|\mathbf{r}-\mathbf{r}^{\prime}\right|\right)=\left(1-\frac{\left|\mathbf{r}-\mathbf{r}^{\prime}\right|^{2}}{l^{2}}\right)
$$

which renders the analytical treatment easier. Very similar results (not shown) were also obtained with other isotropic functions assuming either exponential or Gaussian dependences.

To completely adapt our spatiotemporal random forcing to the kinematic approach, note that, within the spirit of its quasistatic version as it is going to be used here, the spatial structure of the noise will enter only through the successive positions visited by the wandering tip. An exact analytic approach following from such an ansatz is out of our reach, given the complexity of the set of stochastic kinematic equations. So we introduce hereafter a "quasideterministic", approximation by defining effective spatial dependent noises as $\xi(\mathbf{R}(t), t)$ where $\mathbf{R}(t)$ stands for the tip deterministic trajectory, chosen in our case to be represented at its leading order by a circular motion with frequency $\omega_{0}$, corresponding to the unperturbed steadily rotating spiral $[\mathbf{R}(t)$ $\left.=R_{0}\left(\cos \omega_{0} t, \sin \omega_{0} t\right), \mathbf{R}\left(t^{\prime}\right)=R_{0}\left(\cos \omega_{0} t^{\prime}, \sin \omega_{0} t^{\prime}\right)\right]$. Given our success in exploiting the analytic possibilities it offers, this ansatz turns out to be highly rewarding, although one can easily anticipate that such an approximation will break down for spatiotemporal disorder of large correlation times, as will be discussed later on.

\section{SPATIALLY UNIFORM FLUCTUATIONS}

Before addressing the general case of spatiotemporal disorder, we are going to consider fluctuations modeled as pure temporal (spatially uniform) noise. Different ways to proceed can be examined separately depending on the chosen parametric forcing.

\section{A. Fluctuations in the normal velocity for a steady $\left(\omega=\omega_{0}\right)$ rigid rotating spiral $\left(k=k_{c}\right)$}

In the first calculation, rather artificial but useful in illustrating the most important steps of our analytic procedure, we have worked with a system where $\omega=\omega_{0}$ and $k=k_{c}$ are assumed to be constant parameters, while fluctuations are introduced only through $V_{0}$ at the tip equations. This situation could be understood, in fact, as a circular motion of the tip around a core with a fluctuating radius. The set of kinematic equations appropriate to this case reduces to

$$
\begin{gathered}
\frac{d X_{0}}{d t}=-V_{0}(t) \sin \omega_{0} t=-\left[V_{0}+\xi_{V_{0}}(t)\right] \sin \omega_{0} t, \\
\frac{d Y_{0}}{d t}=V_{0}(t) \cos \omega_{0} t=\left[V_{0}+\xi_{V_{0}}(t)\right] \cos \omega_{0} t .
\end{gathered}
$$

Representative trajectories following from these equations are shown in Fig. 3. 

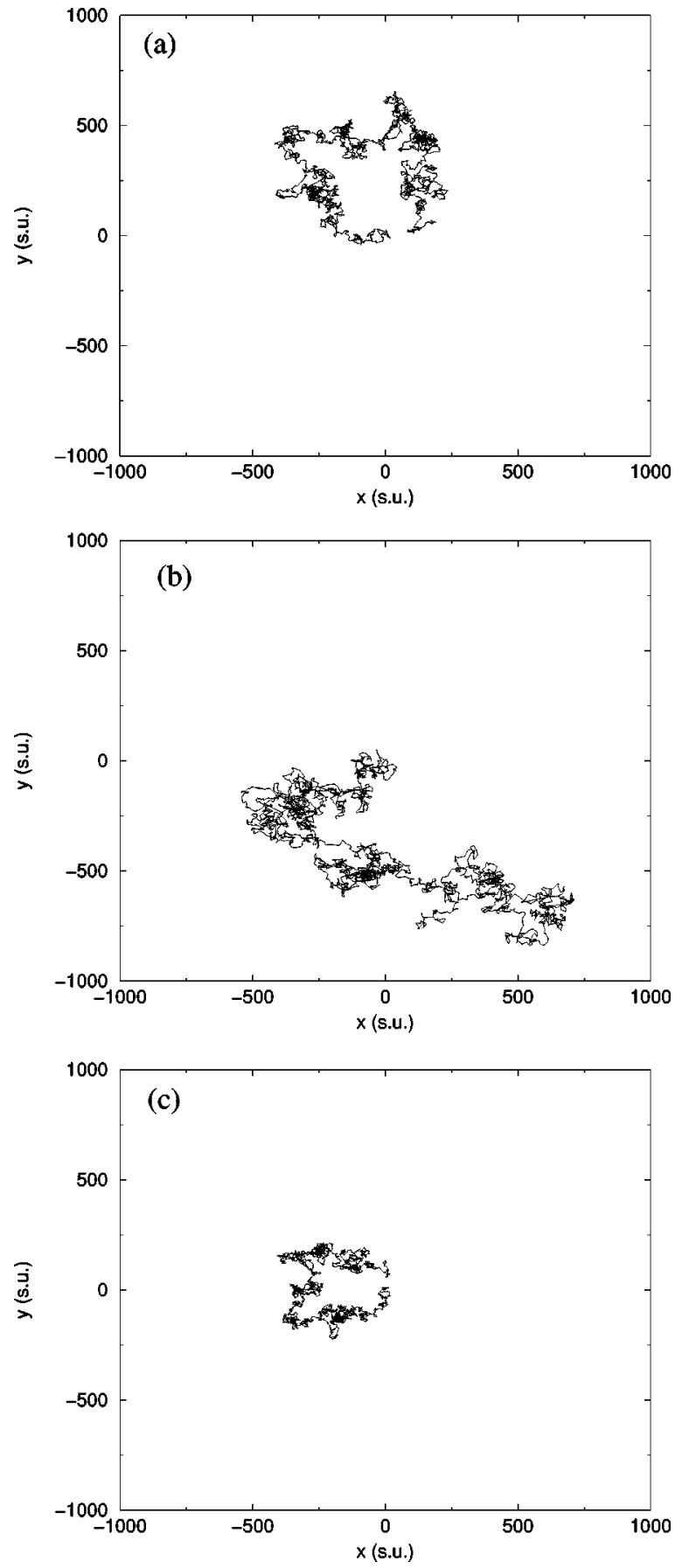

FIG. 3. Numerical simulations of trajectories corresponding to the spiral tip randomly forced according to Eqs. (16) - (17), with different correlation times ( $\tau=10$ t.u., $\tau=50$ t.u., $\tau=500$ t.u.). For simplicity the displacements depicted in this and related following figures actually refer to the center of the spiral core. The spiral parameters are $V_{0}=1, k_{c}=0.1, D=1, \gamma=1.5, R_{0}=46.16$ s.u., $T_{0}$ $=290$ t.u., and $\tau_{g}=30.80$ t.u. Simulations are run for a total time of $3000 T_{0}$. The fluctuations in $V_{0}$ are introduced with $\sigma_{V_{0}}^{2}=0.0025$.

The explicit calculation of a diffusion coefficient accounting for the tip wandering is then exact and particularly straightforward. We start by defining the complex quantity for the tip position $Z=X+i Y$. Then we can rewrite Eqs. (16)-(17) as,

$$
\frac{d Z}{d t}=\left[V_{0}+\xi_{V_{0}}(t)\right] i e^{i \omega_{0} t}
$$

whose formal resolution leads to

$$
Z=\frac{V_{0}}{\omega_{0}} e^{i \omega_{0} t}+i \int_{0}^{t} \xi_{V_{0}}\left(t^{\prime}\right) e^{i \omega_{0} t^{\prime}} d t^{\prime}
$$

A linear time dependence of the squared dispersion of the spiral tip is a signature of Brownian motion, $\left\langle Z^{2}\right\rangle=2 d D t$, characterized by a diffusion coefficient defined as

$$
2 d D=\left\langle\frac{d|Z|^{2}}{d t}\right\rangle_{t \rightarrow \infty}=2 \operatorname{Re}\left(\left\langle\frac{d Z}{d t} Z^{*}\right\rangle\right)_{t \rightarrow \infty},
$$

with $d=2$ for our two-dimensional simulations. So, the problem of the calculation of the diffusion coefficient reduces to the calculation of the average $\left\langle d Z / d t Z^{*}\right\rangle$

$$
\operatorname{Re}\left(\left\langle\frac{d Z}{d t} Z^{*}\right\rangle\right)=\int_{0}^{t}\left\langle\xi_{V_{0}}(t) \xi_{V_{0}}\left(t^{\prime}\right)\right\rangle \cos \omega_{0}\left(t-t^{\prime}\right) d t^{\prime}
$$

Invoking the stationary character of the fluctuations,

$$
\left\langle\frac{d|Z|^{2}}{d t}\right\rangle_{t \rightarrow \infty}=P_{\xi_{V_{0}}}^{\cos }\left(\omega_{0}\right)
$$

where $P_{\xi}^{\cos }\left(\omega_{0}\right)$ stands for the power spectrum of the noise at the characteristic frequency of the rotating spiral

$$
P_{\xi}^{\cos }(\Omega) \equiv \int_{-\infty}^{\infty} C_{\xi}(t) e^{i \Omega t} d t=2 \int_{0}^{\infty} C_{\xi}(t) \cos \Omega t d t
$$

The final expression for the diffusion coefficient then simply reads

$$
D=\frac{1}{4} P_{\xi_{V_{0}}}^{\cos }\left(\omega_{0}\right)
$$

If we choose as a particular noise the Ornstein-Uhlenbeck forcing, this general expression reduces to

$$
D=\frac{\sigma_{V_{0}}^{2}}{2} \frac{\tau}{1+\omega_{0}^{2} \tau^{2}} .
$$

The simulation results in Fig. 4 perfectly agree with the theoretical calculation. Up to this point the treatment is exact, so numerical results in this section are also useful as a benchmark calculation to check our simulations routines.

What is important in Fig. 4 is the resonant effect of the diffusion coefficient when the time scale of the noise, parametrized by its correlation time, matches the characteristic time scale of the spiral rotation parametrized by the inverse of its rotation frequency $\tau_{\max }=\omega_{0}^{-1}=T_{0} / 2 \pi$. Remarkably enough, this important feature of the temporal fluctuations, which was neatly observed in the corresponding BZ experi- 


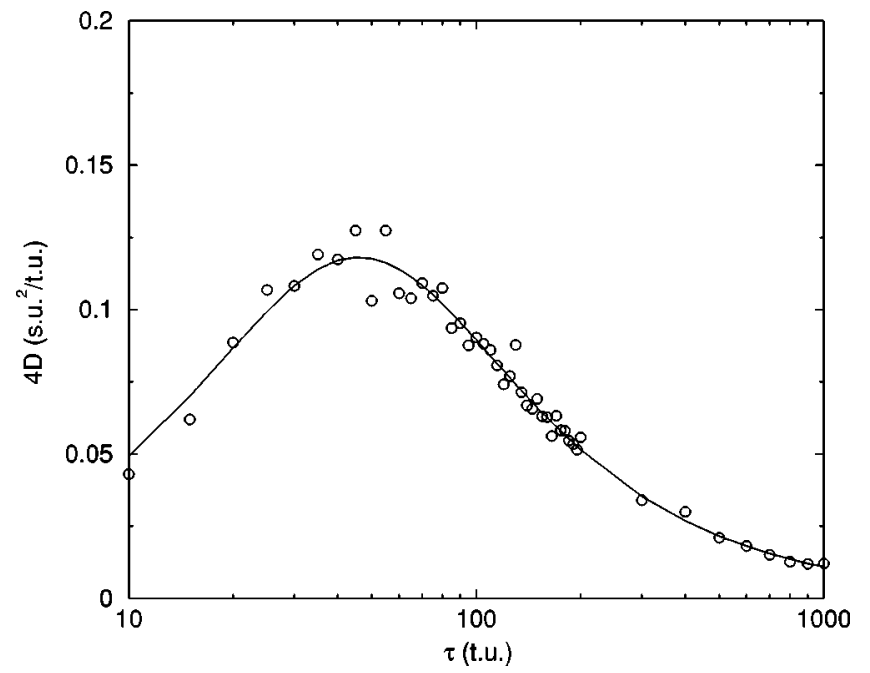

FIG. 4. Numerical results for the effective diffusion coefficient $D$ vs $\tau$, following from Eqs. (16)-(17) for the tip motion with an average of 300 realizations for each correlation time. The spiral parameters are those of Fig. 3 with $\sigma_{V_{0}}^{2}=0.0025$ and $\omega_{0}=0.0216$. Simulations are run for a total time of $10^{4} T_{0}$. The continuous curve fit corresponds to the analytical result given by Eq. (25) with $\sigma_{V_{0 \text { num }}}^{2}=0.0026$ and $\omega_{0 \text { num }}=0.0218$.

ments as recalled in the Introduction, appear to be so robust that it is already captured at the simple level of description proposed so far.

\section{B. Fluctuations on the normal and tangential velocities for a steady $\left(\omega=\omega_{0}\right)$ nonrigid rotating spiral $\left(k \neq k_{c}\right)$}

To the preceding situation, we now add fluctuations introduced through the parameter $k_{c}$, keeping $\omega$ and this time and also $\tau_{g}$ constant. The set of kinematic equations are expressed now as

$$
\begin{gathered}
\frac{d X_{0}}{d t}=-V_{0}(t) \sin \omega_{0} t+\gamma\left[k(t)-k_{c}(t)\right] \cos \omega_{0} t, \\
\frac{d Y_{0}}{d t}=V_{0}(t) \cos \omega_{0} t+\gamma\left[k(t)-k_{c}(t)\right] \sin \omega_{0} t, \\
\frac{d k}{d t}=-\frac{1}{\tau_{g}}\left[k(t)-k_{c}(t)\right] .
\end{gathered}
$$

A formal resolution of Eq. (28) is first needed to define the formal noise acting on the tip's curvature $k$, denoted $\psi(t)$, which can then be introduced into Eqs. (26)-(27), leading similarly as before to

$$
\frac{d Z}{d t}=\left[V_{0}+\xi_{V_{0}}(t)\right] i e^{i \omega_{0} t}+\psi(t) e^{i \omega_{0} t}
$$

In analogy with Eq. (23), we define this time the complementary quantity $P_{C}^{\sin }(\Omega)$, as

$$
P_{\xi}^{\sin }(\Omega) \equiv 2 \int_{0}^{\infty} C_{\xi}(t) \sin \Omega t d t,
$$

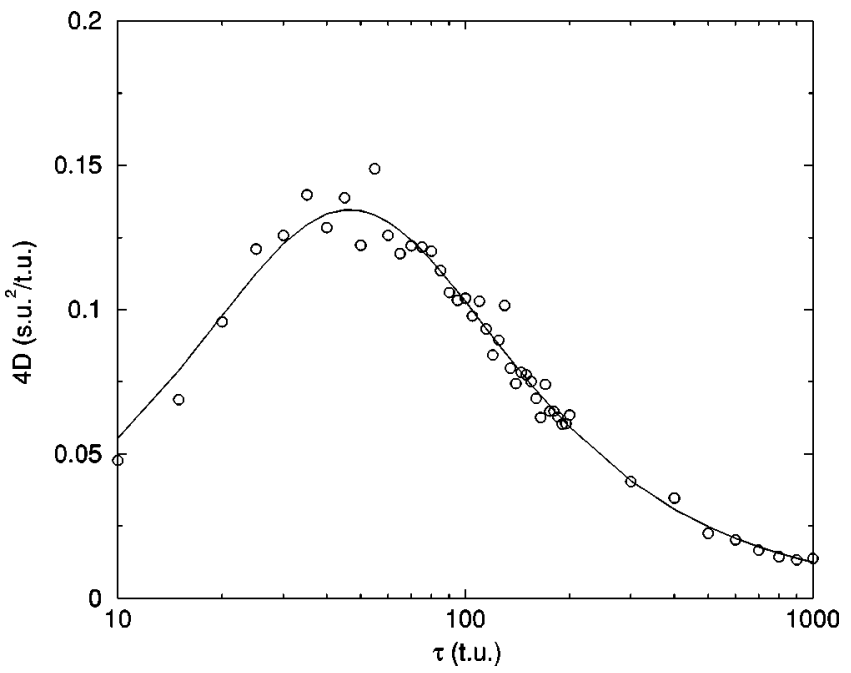

FIG. 5. Numerical results for the effective diffusion coefficient $D$ vs $\tau$ following from Eqs. (26)-(28) for the tip motion with an average of 300 realizations for each correlation time. The spiral parameters are those of Fig. 3 with $\sigma_{V_{0}}^{2}=0.0025$ and $\omega_{0}=0.0216$, and $\nu=0.1$ and $\sigma_{V_{0} k_{c}, m}^{2}=0.0286$ as calculated from Eq. (33). Simulations are run for a total time of $10^{4} T_{0}$. The continuous curve fit corresponds to the analytical result given by Eq. (32) with $\sigma_{V_{0} k_{c}, m, \text { num }}^{2}=0.0290$ and $\omega_{0 \text { num }}=0.0216$.

to arrive to a final result analogous to Eq. (24) above

$$
D=\frac{1}{4}\left[P_{\xi_{V_{0}}}^{\cos }\left(\omega_{0}\right)+P_{\psi}^{\cos }\left(\omega_{0}\right)+P_{\psi \xi_{V_{0}}}^{\sin }\left(\omega_{0}\right)\right] .
$$

For the particular case of the Ornstein-Uhlenbeck statistics, the left averages can be explicitly calculated and put together into a general expression for the diffusion coefficient. The result is totally similar to the previous Eq. (25), but now written in terms of a mixed noise intensity,

$$
D=\frac{\sigma_{V_{0} k_{c}, m}^{2}}{2} \frac{\tau}{1+\omega_{0}^{2} \tau^{2}}
$$

where the exact result for $\sigma_{V_{0} k_{c}, m}^{2}$ reads

$$
\sigma_{V_{0} k_{c}, m}^{2}=\sigma_{V_{0}}^{2}\left(1+\gamma^{2} \nu^{2} \frac{\omega_{0}^{2} \tau_{g}^{2}}{1+\omega_{0}^{2} \tau_{g}^{2}}+2 \gamma \nu \frac{\omega_{0} \tau_{g}}{1+\omega_{0}^{2} \tau_{g}^{2}}\right)
$$

Numerical results for the diffusion coefficient following from simulations of Eqs. (26)-(28) are shown in Fig. 5, which again show perfect agreement between analytical and numerical results.

One particular simplification of this result corresponds to retaining fluctuations on $k_{c}$ only ( $V_{0}=$ constant). In this case there are no fluctuations in $V_{0}$ and the final result reduces simply to

$$
D=\frac{\sigma_{V_{0}}^{2} \gamma^{2} \nu^{2}}{2} \frac{\omega_{0}^{2} \tau_{g}^{2}}{1+\omega_{0}^{2} \tau_{g}^{2}} \frac{\tau}{1+\omega_{0}^{2} \tau^{2}}
$$




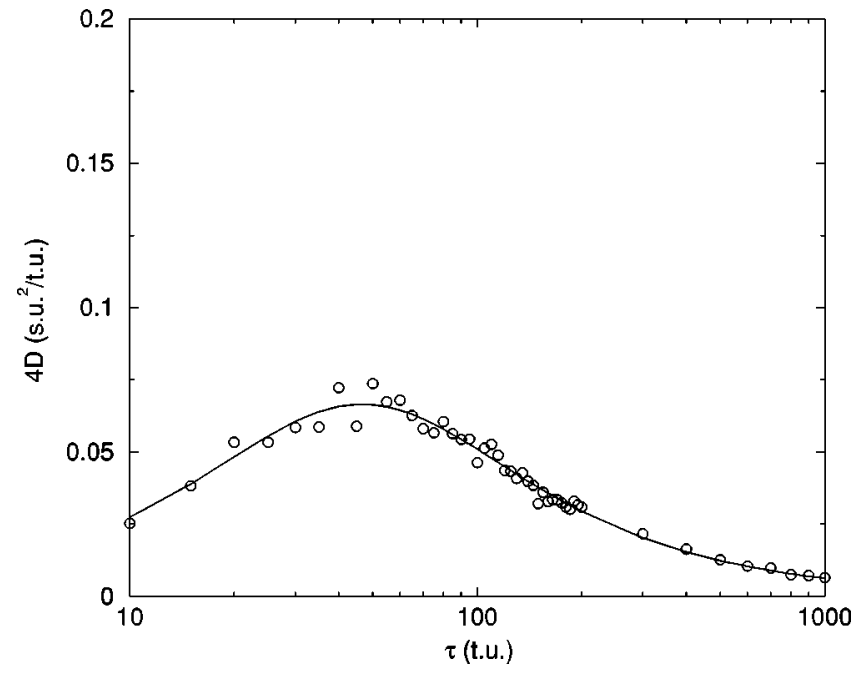

FIG. 6. Numerical results for the effective diffusion coefficient $D$ vs $\tau$ following from the full stochastic equations for the tip motion with an average of 300 realizations for each correlation time. The spiral parameters are those of Fig. 3 with $\sigma_{V_{0}}^{2}=0.0025, \omega_{0}$ $=0.0216$, and $\nu=0.1$. Simulations are run for a total time of $10^{4} T_{0}$. The continuous curve fit corresponds to the effective result given by Eq. (35) with $\sigma_{\text {eff }}^{2}=0.00143$ and $\omega_{0 \text { num }}=0.0215$.

once again with the same $\tau$ dependence but with another prefactor.

\section{Fully stochastic equations $\left(\omega, \tau_{g}\right.$ nonconstant and $\left.k \neq k_{c}\right)$}

The next natural step in our analysis consists in including fluctuations in the kinematic equations but now not only explicitly in the tip equations but wherever the parameters $V_{0}$ and $k_{c}$ appear, i.e., also in the implicit expressions for $\omega$ and $\tau_{g}$.

No analytic calculations are available for this situation, because of the great complexity of the analyses involved. However, for Ornstein-Uhlenbeck noise and according to the previous cases, we expect the same formal result to hold, expressing this time in terms of an effective noise intensity $\sigma_{\mathrm{eff}}^{2} \propto \sigma_{V_{0}}^{2}$

$$
D=\frac{\sigma_{\mathrm{eff}}^{2}}{2} \frac{\tau}{1+\omega_{0}^{2} \tau^{2}} .
$$

To confirm this conjecture we have conducted simulations for different noise realizations and several correlation times. The corresponding results for $D$ are shown in Fig. 6, fully confirming our ansatz.

All the results in this section can be summarized by saying that a neat resonantlike dependence of $D$ with $\tau$ expressed by the maximum at $\tau=\omega_{0}^{-1}$ is obtained in full agreement with experimental results. The physical interpretation of this behavior is as follows. At fixed noise dispersion $\sigma^{2}$, the effect of random perturbations must disappear for vanishing $\tau$ since fast bounded fluctuations will be averaged out by the system. On the other hand for $\omega_{0} \tau \gg 1$, the spiral cores lose mobility because the noise does not change appreciably during a rotation period of the tip. The effect of temporal, spatially uniform noise is thus most effective when noise variations match the intrinsic time scale of the rotating spiral.

\section{SPATIOTEMPORAL FLUCTUATIONS}

Now according to what was anticipated in Sec. IV, the fluctuating parameters are going to depend on the tip position and time $V_{0}=V_{0}(X, Y, t)$ and $k_{c}=k_{c}(X, Y, t)$. The quasideterministic circular trajectory is parametrized as

$$
Z(t)=\frac{V_{0}}{\omega_{0}} e^{i \omega_{0} t}
$$

so that

$$
\left|\mathbf{R}(t)-\mathbf{R}\left(t^{\prime}\right)\right|^{2}=2 \frac{V_{0}^{2}}{\omega_{0}^{2}}\left[1-\cos \omega_{0}\left(t-t^{\prime}\right)\right] .
$$

As we have anticipated, we are going to use such a quasideterministic trajectory to approximate the spatial correlation function, which according to Eq. (15) reads

$$
C\left(\left|\mathbf{r}-\mathbf{r}^{\prime}\right|\right)=\left(1-2 \frac{V_{0}^{2}}{\omega_{0}^{2} l^{2}}\left[1-\cos \omega_{0}\left(t-t^{\prime}\right)\right]\right)
$$

Noise-distributed effects introduced though $V_{0}$ or $k_{c}$ are quite different from those of the case with spatially uniform fluctuations, as will become clear in what follows. For that reason we treat them separately in the next two sections. Note in this respect that the level of approximation in Sec. VIB is different from that in Sec. VB, since here fluctuations will only appear on $k_{c}$ whereas there they entered into both $V_{0}$ and $k_{c}$.

\section{A. Fluctuations in the normal velocity for a steady $\left(\omega=\omega_{0}\right)$ rigid rotating spiral $\left(k=k_{c}\right)$}

As we have done for the temporal case, we are going to begin with the simplest situation, i.e., a circular motion with a fluctuating radius. Similarly to Eq. (18),

$$
\frac{d Z}{d t}=\left[V_{0}+\xi_{V_{0}}(Z, t)\right] i e^{i \omega_{0} t} .
$$

To solve this stochastic differential equation, we proceed as we did in the temporal case [see Eq. (19)], invoking this time the conditions of Eq. (38) for the noise correlation. Following the same formal steps as we did before, we arrive at

$$
\begin{aligned}
\left\langle\frac{d|Z|^{2}}{d t}\right\rangle_{t \rightarrow \infty}= & 2 \int_{0}^{\infty} d t C_{\xi}(t) \cos \omega_{0} t \\
& \times\left(1-\frac{4 V_{0}^{2}}{\omega_{0}^{2} l^{2}} \frac{1}{2}+\frac{4 V_{0}^{2}}{\omega_{0}^{2} l^{2}} \frac{1}{2} \cos \omega_{0} t\right) .
\end{aligned}
$$




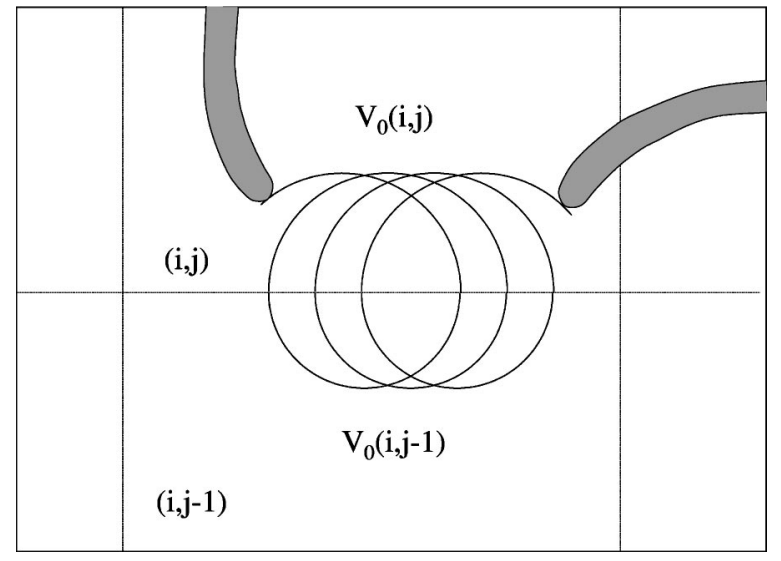

FIG. 7. Boundary effects on spiral motion corresponding to the normal velocity dependence on spatial fluctuations. For the sake of simplicity, the picture corresponds to the limit of frozen noise (infinite $\tau$ ). The tip motion is supposed to begin at the left on the pixel $[i, j]$ where it rigidly rotates with a characteristic radius. After halfperiod it crosses to the next noise pixel $[i, j-1]$, where the velocity and in turn the rotation radius are different. After completing another half-period the tip has gained a net displacement of $2\left[R_{0}(i, j-1)-R_{0}(i, j)\right]$ per rotating period. This effect would repeat for the next period.

The diffusion coefficient can thus be expressed as a function of the power spectrum of the noise at the characteristic frequency and multiples of this frequency

$D=\frac{P_{\xi_{V_{0}}}^{\cos }\left(\omega_{0}\right)}{4}+\frac{V_{0}^{2}}{4 \omega_{0}^{2} l^{2}}\left[P_{\xi_{V_{0}}}^{\cos }(0)-2 P_{\xi_{V_{0}}}^{\cos }\left(\omega_{0}\right)+P_{\xi_{V_{0}}}^{\cos }\left(2 \omega_{0}\right)\right]$.

For the Ornstein-Uhlenbeck noise this formal expression turns into

$$
D=\frac{\sigma_{V_{0}}^{2}}{2} \frac{\tau}{1+\omega_{0}^{2} \tau^{2}}+\frac{\sigma_{V_{0}}^{2}}{2} \frac{V_{0}^{2}}{\omega_{0}^{2} l^{2}}\left(\tau-\frac{2 \tau}{1+\omega_{0}^{2} \tau^{2}}+\frac{\tau}{1+4 \omega_{0}^{2} \tau^{2}}\right) .
$$

Note that this last expression, compared with the corresponding result, Eq. (25), for spatially uniform fluctuations, correctly predicts an enhancement of the spiral dispersion due to the spatiotemporal character of the random forcing. However, the unbounded quasilinear growth of $D$ with $\tau$, which appears in Eq. (42), is an artifact of our quasideterministic approximation to the correlation function that will be corrected later on. Before doing that, it is interesting to examine the dispersion trajectories following to the stochastic dynamics prescribed by Eq. (39). As shown qualitatively in Fig. 7, the core diffuses temporally until it finds a boundary between noise cells. Then, for large enough $\tau$ the tip does one part of the rotation motion with one velocity and another part with another velocity. If both velocities are different, which is likely to occur since noise realizations are uncorrelated in each cell, the radius will be different too. The final result is that there is a net transversal velocity experienced by the tip and a corresponding lateral drift of the spiral core in its attached motion to the boundaries of the noise cells. Large
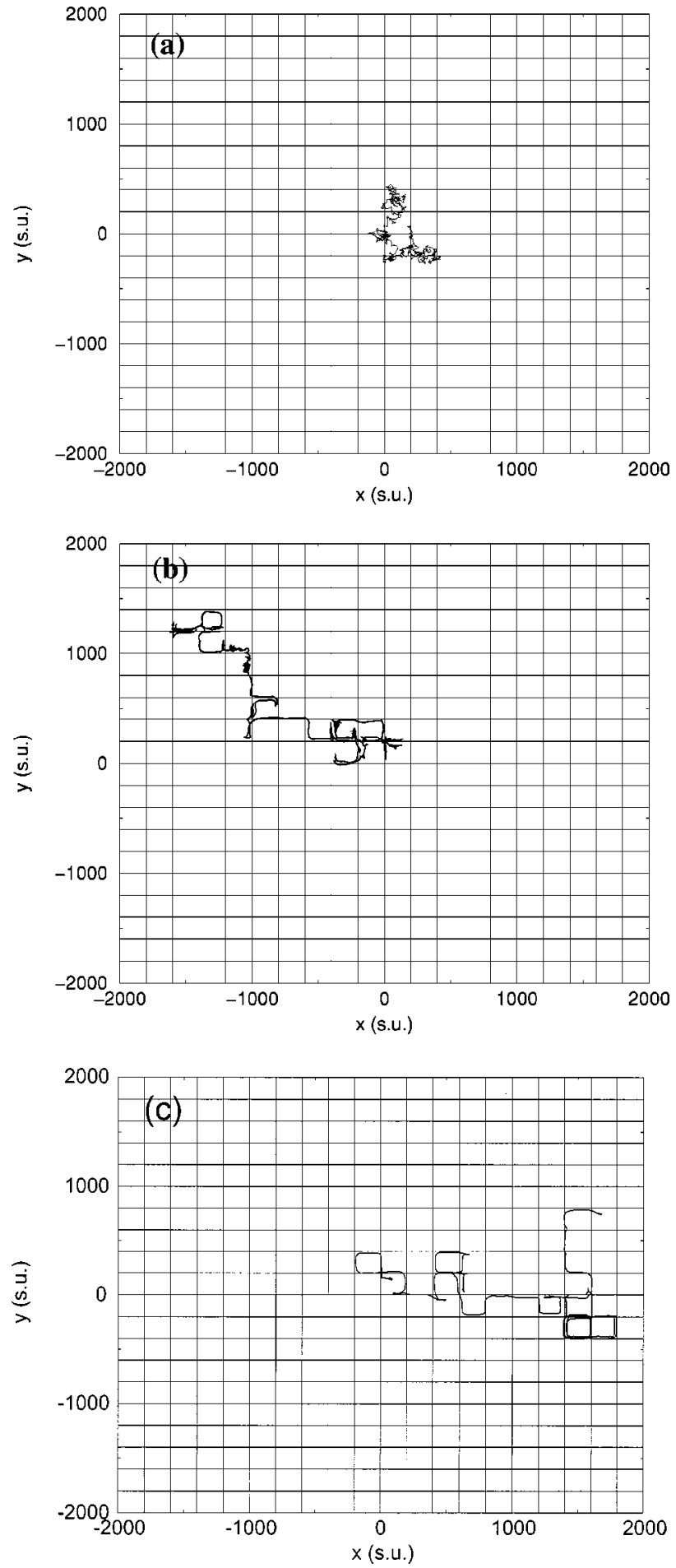

FIG. 8. Numeric simulations of trajectories corresponding to the spiral tip randomly forced according to Eq. (39), with different correlation times ( $\tau=50$ t.u., $\tau=1000$ t.u., $\tau=10000$ t.u. $)$ and a correlation length of $l=200$ s.u. The spiral parameters are those of Fig. 3. Simulations are run for a total time of $300 T_{0}$. The fluctuations in $V_{0}$ are introduced with $\sigma_{V_{0}}^{2}=0.0225$.

scale numeric simulations shown in Fig. 8 reproduce this motion mode, which indeed turns out to be a very effective dispersion mechanism for spiral dispersion singularly for large values of $\tau$. 


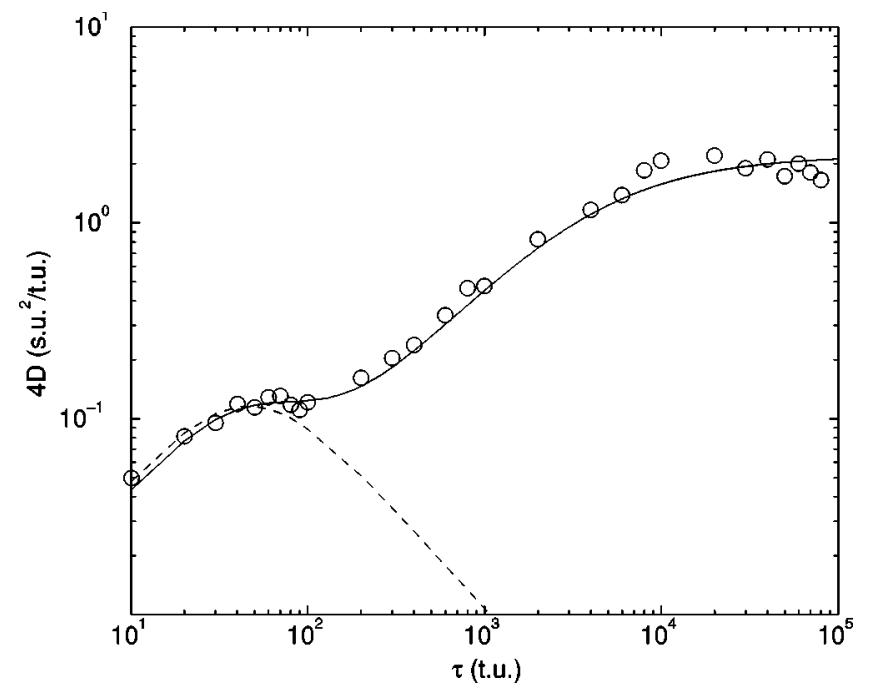

FIG. 9. Numeric results for the effective diffusion coefficient $D$ vs $\tau$ following from Eq. (39) for the tip motion, with an average of 200 realizations for each correlation time. The spiral parameters are those of Fig. 3, with $\sigma_{V_{0}}^{2}=0.0025$ and $l=200$ s.u. Simulations are run for a total time of $1000 T_{0}$. The continuous curve fit corresponds to Eq. (42) corrected with an effective correlation time [see Eq. (43)] with $\sigma_{V_{0}}^{2}=0.0025$, and fitting parameters $V_{0}^{2} / \omega_{0}^{2} l^{2}=0.11$ and $\tau_{\text {eff }}=4000$ t.u. Dashed line, shown for comparison, corresponds to the case of spatially uniform fluctuations.

Needless to say and in view of the tip trajectories we have just described, it would have been rather surprising that our quasideterministic ansatz would have been able to reproduce the numeric results for correlation times larger than typically the rotation period of the spiral. Actually, by proposing an ansatz based on a deterministic closed motion of the tip, we introduce spurious effects at large $\tau$, since the effective noise realizations experienced by the tip appear strongly correlated after every rotation period. In fact, the actual trajectory is not closing in itself and decorrelates the effective noise by exploring spatially uncorrelated regions.

The most reasonable way to overcome this limitation is by introducing a cutoff of the correlation times. This amounts to replacing $\tau$ by $\tau^{\prime}$, defined as

$$
\frac{1}{\tau^{\prime}} \equiv \frac{1}{\tau}+\frac{1}{\tau_{\mathrm{eff}}},
$$

in terms of the ad hoc parameter $\tau_{\text {eff }}$, interpreted as the time needed for the tip to cross over a pixel, and in what follows considered as a fitting parameter. Proceeding in this way, analytical and numerical results are perfectly comparable, as shown in Fig. 9.

\section{B. Fluctuations in the tangential velocity for a steady $\left(\omega=\omega_{0}\right)$ nonrigid rotating spiral $\left(k \neq k_{c}\right)$}

Numeric simulations corresponding to this situation are shown in Fig. 10. They are indeed very different as compared to those presented in the last section as a clear manifestation of the singular effect of the spatiotemporal random forcing when allowed to act on the sprouting or contracting motion of the spiral. A close examination of the tip motion
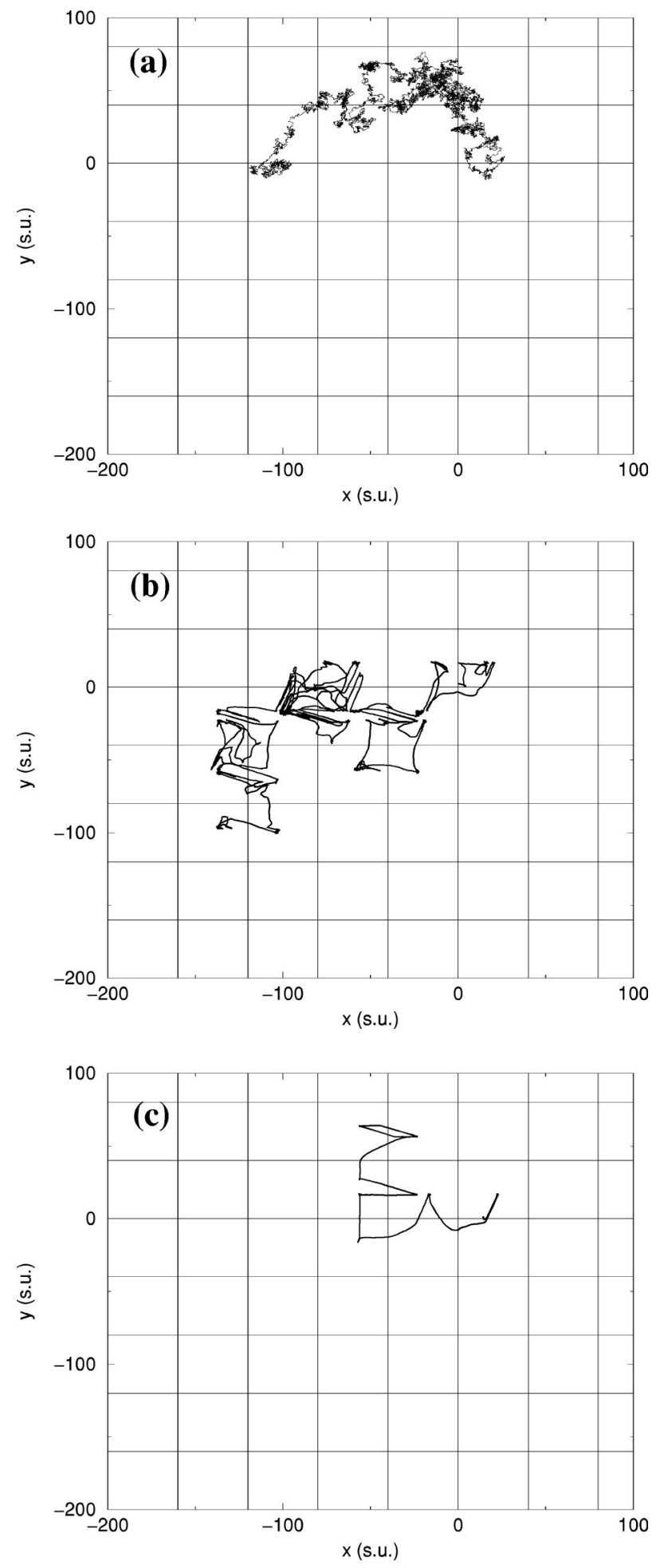

FIG. 10. Numerical simulations of trajectories corresponding to fluctuations on the tangential velocity of the tip, with different correlation times ( $\tau=20$ t.u., $\tau=2000$ t.u., $\tau=200000$ t.u.) and a correlation length of $l=40 \mathrm{~s}$.u. The spiral parameters have been changed to increase $\tau_{g}$ to better see the pinning effect (see text): $V_{0}=1$, $k_{c}=0.2, \quad D=1, \quad \gamma=0.4, \quad R_{0}=16.32$ s.u., $T_{0}=102$ t.u., and $\tau_{g}$ $=40.8$ t.u. Simulations are run for a total time of $15000 T_{0}$. The fluctuations in $k_{c}$ are introduced with $\sigma_{V_{0}}^{2}=0.25$ and $\nu=0.1$. 

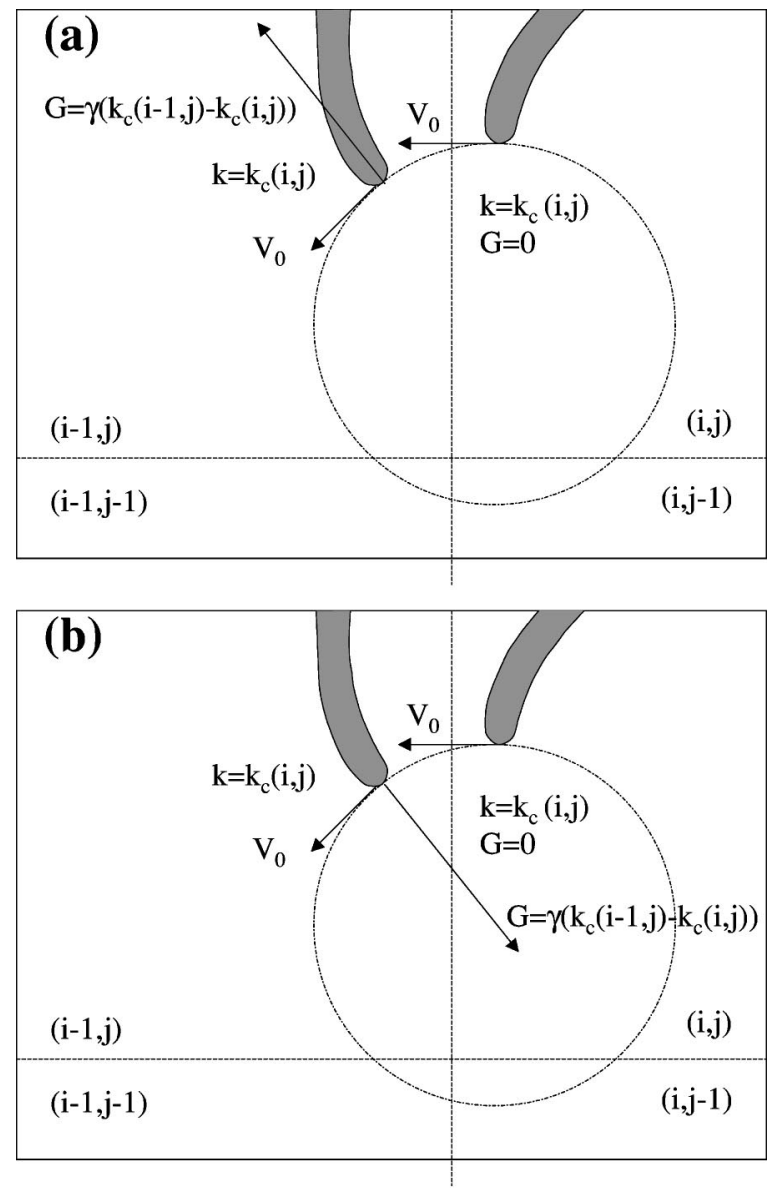

FIG. 11. Boundary effects for the curvature dependence on the fluctuations forcing the spiral motion. The tip is shown to rotate rigidly $\left[k=k_{c}(i, j)\right]$ on the pixel $[i, j]$ with no tangential velocity. When it crosses the boundary, the value for $k_{c}$ is updated to $k_{c}(i-1, j)$ but the instantaneous tip curvature must evolve from the previous value $k_{c}(i, j)$. A tangential velocity appears right after crossing, $G=\gamma\left[k_{c}(i-1, j)-k_{c}(i, j)\right]$, which is positive (negative) if $k_{c}(i-1, j)>(<) k_{c}(i, j)$ as we can see on the first (second) picture.

reveals that it quasisteadily rotates inside a cell until a boundary is found when the tip chooses either to remain in the same pixel, after which the boundary sends the core back, or to leave it very quickly. The tip decides its behavior depending on the relation between the pair of values of $k_{c}$ in the two adjacent pixels.

This behavior can be easily interpreted since when crossing a boundary the value of $k_{c}$ changes instantaneously, but the change in $k$ is not completely adiabatic, as dictated by the characteristic relaxation time $\tau_{g}$ (provided this time constant is large enough). Thus we do have a non-null and stochastically varying tangential velocity for the tip given by $G=\gamma\left(k_{c}-k\right)$. Then if $k_{c}>k\left(k_{c}<k\right)$, there is a positive (negative) tangential velocity, and the tip returns to (goes straight to) the previous (next) pixel [see Fig. 11].

As a matter of fact, this behavior closely resembles a sort of pinned motion, according to which the tip remains confined within a cell for long-time intervals, interrupted by very fast episodes when crossing the pixel boundaries. Actu-

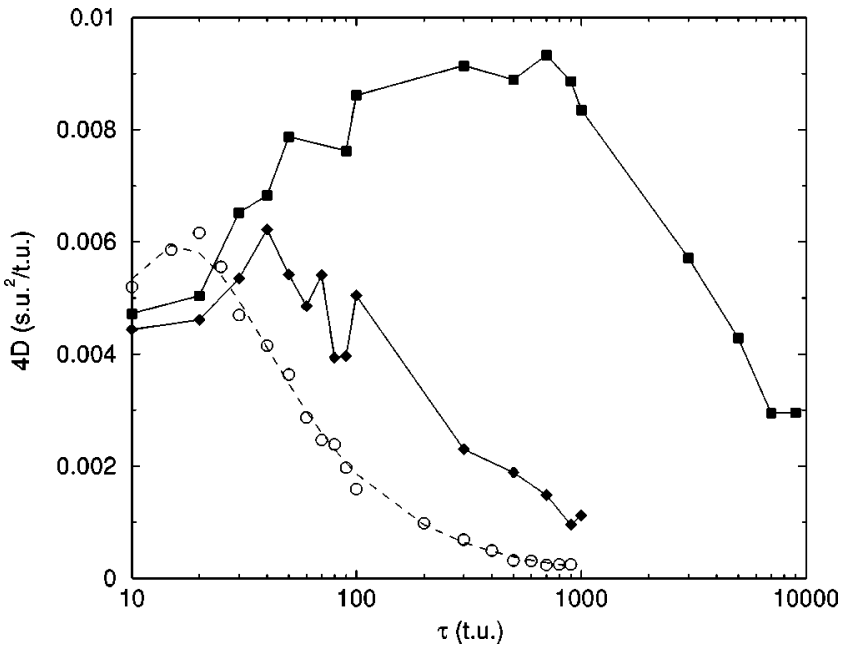

FIG. 12. Numerical results for the effective diffusion coefficient $D$ vs $\tau$, following from fluctuations through the tangential velocity. The spiral parameters are those of Fig. 10 with $\sigma_{V_{0}}^{2}=0.25, \nu=0.1$, and $l=40$ s.u. (squares), with an average of 200 realizations for each correlation time, and $l=60 \mathrm{~s}$.u. (diamonds), with an average of 50 realizations. Simulations are run for a total time of $20000 T_{0}$. Dashed line, shown for comparison, corresponds to the case of spatially uniform fluctuations.

ally, this pinning effect enables us to easily interpret the characteristic behavior of the diffusion coefficient for long correlation times $(D \propto 1 / \tau)$ [see Fig. 12]. A simple argument goes as follows. For large $\tau$ the dispersion motion inside a pixel, as represented by Eq. (34), corresponds to a time lag, for fixed $l$, proportional to $\tau\left[l^{2} \simeq 2 \tau /\left(1+\omega_{0}^{2} \tau^{2}\right) t\right]$. Faster transitions between pixels, when they correspond, take a very short time. So essentially the tip dispersion increases by fixed amounts of order $l^{2}$ in time steps proportional to $\tau$.

As we can see in Fig. 12 such a pinned motion drastically reduces the spiral dispersion for large $\tau$, the effect being more pronounced for larger pixel size. Obviously, tip anchoring is longer lasting for larger pixels where the tip can rotate without the presence of the pixel boundaries. When the pixel size is smaller the tip is forced to move across the pixel boundaries more often leading to some enhanced dispersion. We finally emphasize that since the curvature relaxation time is set by the parameter $\tau_{g}$, the pinning effect is more effective as this value increases. Furthermore by comparing globally the results in Fig. 12 with those in Fig. 9 we see that the values of the diffusion coefficient under spatiotemporal and spatially uniform fluctuations in $k_{c}$ are similar, whereas in Fig. 9, when considering fluctuations in $V_{0}$, the diffusion coefficient under spatiotemporal fluctuations is typically one order of magnitude larger than for spatially uniform noise.

\section{Fully stochastic equations $\left(\omega, \tau_{g}\right.$ nonconstant and $\left.k \neq k_{c}\right)$}

In this last section we are going to consider the most general scenario retaining spatiotemporal fluctuations in both $V_{0}=V_{0}(X, Y, t)$ and $k_{c}=k_{c}(X, Y, t)$. It is quite reasonable to expect mixed trends of spiral dispersion combining the two distinctive modes just mentioned. This is indeed the 

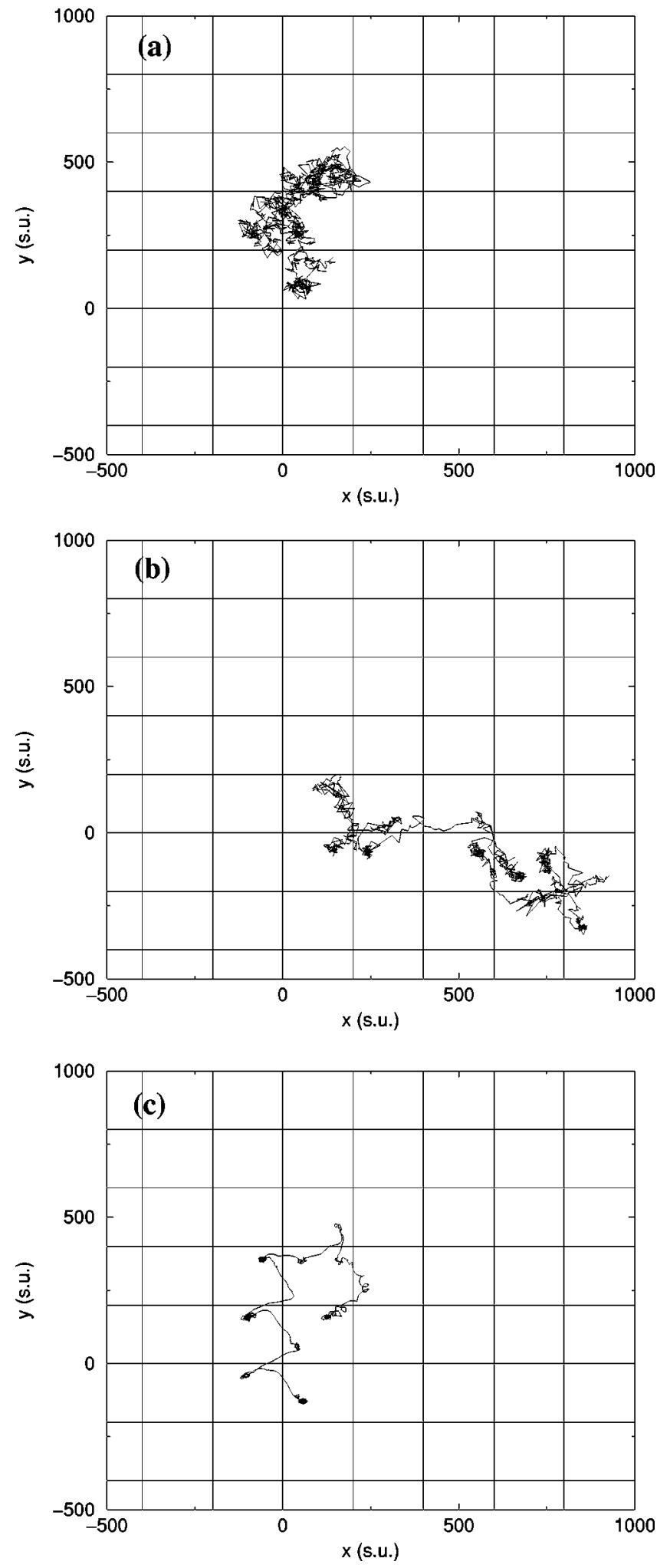

FIG. 13. Numerical simulations of trajectories corresponding to the full stochastic scheme, with different correlation times $(\tau$ $=100$ t.u., $\tau=1000$ t.u., $\tau=30000$ t.u.) and a correlation length of $l$ $=200$ s.u. The spiral parameters are those of Fig. 3. Simulations are run for a total time of $1000 T_{0}$. The fluctuations in $V_{0}$ and $k_{c}$ are introduced with $\sigma_{V_{0}}^{2}=0.0225$ and $\nu=0.1$.

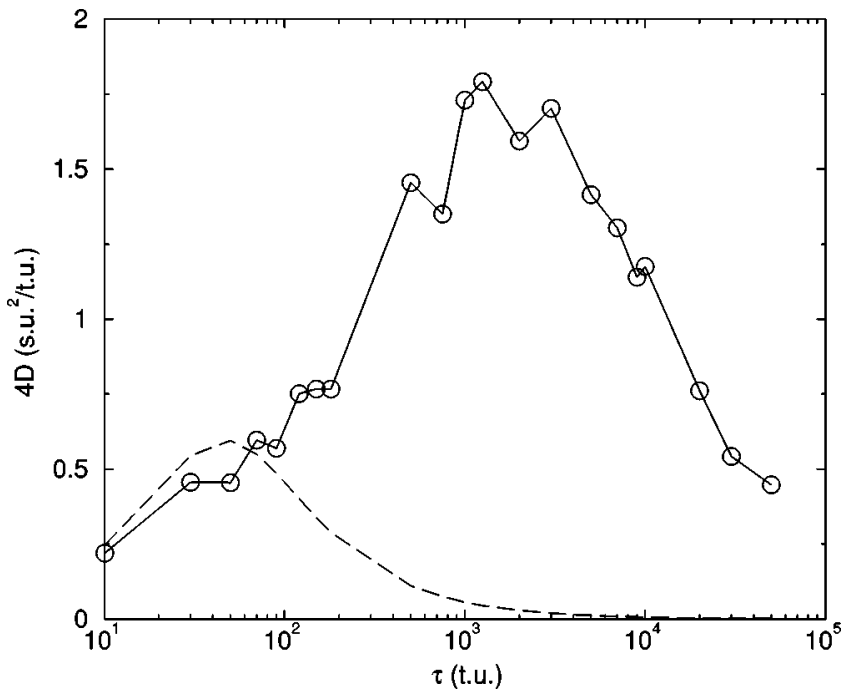

FIG. 14. Numerical results for the effective diffusion coefficient $D$ vs $\tau$ following from the full stochastic equations for the tip motion with an average of 200 realizations for each correlation time. The spiral parameters are those of Fig. 3 with $\sigma_{V_{0}}^{2}=0.0225$ and $l=100$ s.u. Simulations are run for a total time of $1000 T_{0}$. Dashed line, shown for comparison, corresponds to the case of spatially uniform fluctuations.

case as depicted by the set of representative trajectories shown in Fig. 13. If we accept, as proposed here following the results quoted in Sec. IV, that the relative values of the fluctuations in both parameters are of the same order, one can easily conclude that the dominance of each mode will essentially depend on the value of the correlation time relative to the time scale of the curvature relaxation. In the limit of very small values of $\tau$, the spatiotemporal structure of the noise is going to be irrelevant, given the pointlike, i.e., purely tip based, description of the spiral motion at the origin of the quasistatic version of the kinematic approach employed here. For larger values of $\tau$, but still smaller than $\tau_{g}$, the effects of random forcing on the normal velocity are dominant and the dispersion of the spiral is very effective as dictated by the erratic motion of the tip attached to the pixel boundaries. Finally, when increasing $\tau$ and exceeding $\tau_{g}$ we expect a crossover to a pinnedlike much less effective dominated dispersion. These qualitative predictions fully explain the numerical results summarized in Fig. 14.

\section{CONCLUSIONS}

We have systematically examined, analytically and by means of numerical simulations, the behavior of spiral excitation waves forced with spatiotemporal random forcing. Our initial motivation was the related experiments we had conducted with the light-sensitive version of the BZ reaction. The formalism presented here can actually be extended to spiral waves in any excitable system [3-5] under the limit of weak excitability. In any case, the necessary ingredient is to know the relations, such as those in Fig. 2, between the kinematic parameters and the externally controlled excitability. This is so because our approach is based totally on the use of the quasistatic version of the phenomenological kinematic 
theory, which reduces the whole spiral dynamics to the motion of a pointlike object representing its tip.

The statistics considered refers to Ornstein-Uhlenbech forces, although the analytic approach has been generalized as much as possible, especially in the particular and simpler situation of uniform fluctuations (pure temporal forcing). In this case, a neat resonantlike dependence of the diffusion coefficient on the correlation time for fixed noise intensity has been evidenced.

Under the more general scenario corresponding to spatiotemporal disorder, a pair of distinctive dispersion modes have been identified. A very efficient one, represented by an erratic motion attached to the pixel boundaries, results from the fluctuations introduced into the normal velocity of the spiral tip. When the correlation time is increased, a crossover takes place from this behavior to a much less favorably dispersed, pinnedlike motion as follows from the effect of fluctuations on the tangential velocity of the spiral tip.
Following this paper one could imagine either experimental, numerical, or analytical extensions of the problem at hand, aimed particularly at examining the behavior of excitable systems, or spiral supporting related ones, under spatiotemporal random forcing patterns other than those prescribed here.

\section{ACKNOWLEDGMENTS}

Discussions with J. Casademunt, L. Ramírez-Piscina, and J. M. Sancho are acknowledged. We also thank the group of Física no lineal of the University of Santiago de Compostela for providing us with the data of Fig. 1 and particularly to I. Sendina-Nadal for some comments. This work was partially supported by the Comisión Interministerial de Ciencia y Tecnología and Comissionat per a Universitats i Recerca (Generalitat de Catalunya) under Project Nos. PB96-1001 and 1997SGR00090.
[1] Chemical Waves and Patterns, edited by R. Kapral and K. Showalter (Kluwer Academic, Dordrecht, 1993).

[2] A. T. Winfree, Science 175, 634 (1972).

[3] S. Jakubith, H. H. Rotermund, W. Engel, A. von Oertzen, and G. Ertl, Phys. Rev. Lett. 65, 3013 (1990).

[4] F. Siegert and C. J. Weijer, Physica D 49, 224 (1991), and references therein. See also E. Ben Jacob, I. Cohen, and H. Levine, Adv. Phys. 49, 395 (2000) for an updated overview.

[5] J. M. Davidenko, A. M. Pertsov, K. Salomonsz, W. T. Baxter, and J. Jalife, Nature (London) 355, 349 (1991).

[6] K. I. Agladze, V. A. Davydov, and A. S. Mikhailov, Pis'ma Zh. Éksp. Teor. Fiz. 45, 601 (1987) [JETP Lett. 45, 767 (1987)].

[7] O. Steinbock, V. Zykov, and S. C. Müller, Nature (London) 366, 322 (1993).

[8] M. Markus, Z. Nagy-Ungvarai, and B. Hess, Science 257, 225 (1992).

[9] O. Steinbock, J. Schütze, and S. C. Müller, Phys. Rev. Lett. 68, 248 (1992).

[10] S. Grill, V. S. Zykov, and S. C. Müller, Phys. Rev. Lett. 75, 3368 (1995); V. S. Zykov, A. S. Mikhailov, and S. C. Müller, ibid. 78, 3398 (1997); D. M. Goldschmidt, V. S. Zykov, and S. C. Müller, ibid. 80, 5220 (1998).

[11] A. Karma and V. S. Zykov, Phys. Rev. Lett. 83, 2453 (1999).

[12] M. N. Chee, S. G. Whittington, and R. Kapral, Physica D 32, 437 (1988).

[13] M. Bär, I. G. Kevrekidis, H. H. Rotermund, and G. Ertl, Phys. Rev. E 52, R5739 (1995); M. Bär, A. K. Bangia, I. G. Kevrekidis, G. Haas, H. H. Rotermund, and G. Ertl, J. Phys. Chem. 100, 19106 (1996).
[14] M. Vinson, Physica D 116, 313 (1998).

[15] G. V. Osipov, B. V. Shulgin, and J. J. Collins, Phys. Rev. E 58, 6955 (1998).

[16] M. Hendrey, E. Ott, and T. M. Antonsen, Phys. Rev. Lett. 82, 859 (1999); Phys. Rev. E 61, 4943 (2000).

[17] J. Maselko and K. Showalter, Physica D 49, 21 (1991).

[18] J. Wang, S. Kádár, P. Jung, and K. Showalter, Phys. Rev. Lett. 82, 855 (1999).

[19] J. García-Ojalvo and L. Schimansky-Geier, Europhys. Lett. 47, 298 (1999).

[20] P. Jung and G. Mayer-Kress, Phys. Rev. Lett. 74, 2130 (1995).

[21] S. Kádár, J. Wang, and K. Showalter, Nature (London) 391, 770 (1998).

[22] H. Hempel, L. Schimansky-Geier, and J. García-Ojalvo, Phys. Rev. Lett. 82, 3713 (1999).

[23] I. Sendiña-Nadal, A. P. Munuzuri, D. Vives, V. PérezMuñuzuri, J. Casademunt, L. Ramírez-Piscina, J. M. Sancho, and F. Sagués, Phys. Rev. Lett. 80, 5437 (1998).

[24] I. S. Aranson, H. Chaté, and L-H. Tang, Phys. Rev. Lett. 80, 2646 (1998).

[25] V. Pérez-Muñuzuri, F. Sagués, and J. M. Sancho, Phys. Rev. E 62, 94 (2000).

[26] I. Sendiña-Nadal, S. Alonso, V. Pérez-Muñuzuri, M. GómezGesteira, V. Pérez-Villar, L. Ramírez-Piscina, J. Casademunt, J. M. Sancho, and F. Sagués, Phys. Rev. Lett. 84, 2734 (2000).

[27] A. S. Mikhailov, Foundations of Synergetics-Distributed Active Systems (Springer-Verlag, Berlin, 1990); A. S. Mikhailov, D. A. Davydov, and V. S. Zykov, Physica B 70D, 1 (1994).

[28] J. García-Ojalvo and J.M. Sancho, Noise in Spatially Extended Systems (Springer-Verlag, New York, 1999). 